\title{
Biological dose summation of intensity-modulated arc therapy and image-guided high-dose-rate interstitial brachytherapy in intermediate- and high-risk prostate cancer
}

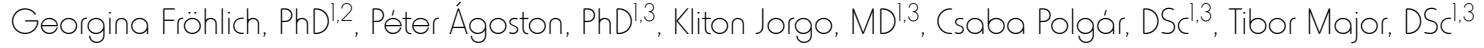 \\ 'Centre of Radiotherapy, National Institute of Oncology, Budapest, Hungary, ${ }^{2}$ Faculty of Science, Eötvös Loránd University, Budapest, \\ Hungary, ${ }^{3}$ Department of Oncology, Faculty of Medicine, Semmelweis University, Budapest, Hungary
}

\begin{abstract}
Purpose: To present an alternative method for summing biologically effective doses of intensity-modulated arc therapy (IMAT) as teletherapy (TT), with interstitial high-dose-rate (HDR) brachytherapy (BT) boost in prostate cancer. Total doses using IMAT boost was compared with BT boost using our method.

Material and methods: Initially, 25 IMAT TT plus interstitial HDR-BT plans were included, and additional plans using IMAT TT boost were created. The prescribed dose was 2/44 Gy to the whole pelvis, 2/60 Gy to the prostate and seminal vesicles, and $1 \times 10$ Gy BT or 2/18 Gy IMAT TT to the prostate. Teletherapy computed tomography (CT) was registered with ultrasound (US) of BT, and the most exposed volume of critical organs in BT were identified on these CT images. The minimal dose of these from IMAT TT was summed with their BT dose, and these $\mathrm{EQD}_{2}$ doses were compared using BT vs. IMAT TT boost. This method was compared with uniform dose conception (UDC).

Results: $\mathrm{D}_{90}$ of the prostate was significantly higher with BT than with IMAT TT boost: 99.3 Gy vs. 77.9 Gy, $p=0.0034$. The $\mathrm{D}_{2}$ to rectum, bladder, and hips were 50.3 Gy vs. $76.8 \mathrm{~Gy}(p=0.0117), 64.7 \mathrm{~Gy}$ vs. 78.3 Gy $(p=0.0117)$, and $41.9 \mathrm{~Gy}$ vs. $50.6 \mathrm{~Gy}(p=0.0044)$, while $\mathrm{D}_{0.1}$ to urethra was $96.1 \mathrm{~Gy}$ vs. $79.3 \mathrm{~Gy}(p=0.0180)$, respectively. UDC overestimated $\mathrm{D}_{2}$ (rectum) by $37 \%(p=0.0117), \mathrm{D}_{2}$ (bladder) by $5 \%(p=0.0214)$, and underestimated $\mathrm{D}_{0.1}$ (urethra) by $1 \%$ $(p=0.0277)$.

Conclusions: Based on our biological dose summation method, the total dose of prostate is higher using BT boost than the IMAT. BT boost yields lower rectum, bladder, and hips doses, but higher dose to urethra. UDC overestimates rectum and bladder dose and underestimates the dose to urethra.

J Contemp Brachytherapy 2020; 12, 3: 260-266 DOI: https://doi.org/10.5114/jcb.2020.96868
\end{abstract}

Key words: prostate cancer, dose summation, integrated biological doses, intensity-modulated arc therapy, interstitial brachytherapy.

\section{Purpose}

The standard of care in curative treatment of intermediate- and high-risk prostate cancer is external beam radiotherapy (teletherapy - TT) and high-dose-rate (HDR) interstitial brachytherapy (BT) boost with androgen deprivation therapy. Since the $\alpha / \beta$ value of prostate tumor is low, dose escalation has an essential role in the development of both radiotherapy modalities [1,2]. The more complex the techniques, the more they can escalate the dose to the tumor while sparing organs at risk (OARs). The state-of-the-art radiotherapy combination is intensity-modulated arc therapy (IMAT) as TT and image-guided interstitial BT $[3,4]$. These complex treatments require reliable reporting of the dose received by tumor and critical structures.

The use of BT boost has been linked with improved biochemical progression-free and overall survival $[5,6]$. What is more, modern HDR-BT approach results in improved quality of life with lower acute urinary and rectal toxicity [7], and with the dose coverage of target volume $\left(\mathrm{D}_{90}\right.$, the minimum dose delivered to $90 \%$ of prostate) correlating with local tumor control [8] and the dose of the most exposed part of OARs with normal tissue toxicity [9].

To achieve reporting these dose-volume parameters properly, overall volumetric doses must be properly integrated with tele- and brachytherapy. As simple physical dose summation does not take into consideration different 
biological effects, the equivalent dose given in 2 Gy fractions $\left(\mathrm{EQD}_{2}\right)$ must be calculated $[10,11]$. The dose distribution of TT is assumed to be completely uniform, so the dose from TT is considered as the target volume, and the nearest OARs receive the entire prescribe dose. Then, this equivalent uniform dose is calculated for dose summation with BT doses (uniform dose conception - UDC) [12]. On the other hand, this assumption can be suitable for conventional four-field box TT plans but an improper approach for IMAT plans, where the dose intensity is modulated. In addition, in the IMAT TT technique, the most exposed $2 \mathrm{~cm}^{3}$ of OARs is not a compact volume, since its voxels are dispersed in organ, as we have reported earlier [13]. It was also shown that the most exposed part of OARs in the summed plans is in the same region that receives the largest dose in BT. Nevertheless, this $2 \mathrm{~cm}^{3}$ volume is not in the same location as the most exposed part in TT [14]. Therefore, simple dose-volume histogram (DVH) addition sums the dose of two different $2 \mathrm{~cm}^{3}$ volumes.

In many previous investigations, authors did not consider the real biological dose of prostate and OARs in TT in combined TT and BT treatment. Pinkawa et al. [15] used the above mentioned UDC method to estimate doses from TT and engaged physical BT doses only. Andrzejewski et al. [16] compared different advanced radiotherapy methods for boosting dominant intraprostatic lesion. They calculated biological equivalent doses for comparison but did not examine combined therapies. Pieters et al. [17] calculated and compared the total doses of $70 \mathrm{~Gy}$, with five-beam intensity-modulated TT combined with 6 Gy TT boost versus 46 Gy TT with HDR or pulsed-dose-rate BT boost complemented to overall $70 \mathrm{~Gy} \mathrm{EQD}_{2}$, but for low-risk patients where the target volume was only the prostate. They redefined the prescribed dose of BT to sum the TT and BT plans in dosimetrically equivalent way. Kikuchi et al. [18] made a computed tomography (CT) series after BT and calculated the biological effective dose of rectum in TT and BT. They associated this dose to the pixels of rectum volume and computed a summarized DVH of TT and BT. This was a better estimation of the rectal dose than the UDC method, but the quadratic behavior of biological dose was not considered. Applying the linear-quadratic formula for a dose-volume parameter is not appropriate, because the $\mathrm{EQD}_{2}$ dose of a voxel is based on the $\alpha / \beta$ value in the given voxel, which is not homogeneous in the body. Therefore, the biological dose must be calculated voxel-by-voxel in the same organ, but currently, none of the treatment planning systems apply this feature.

In the future, the deformable image registration (DIR) could be an appropriate method to integrate BT and TT doses both for prostate and OARs, but at the moment, it results in significant errors at the border of prostate and OARs, i.e. the prostate and the rectal wall overlap each other in registered image. The main problem are foreign bodies (metal or plastic needles and ultrasound [US] probe) in situ, which are not present on TT image data sets. In addition, DIR algorithms cannot handle US data, so CT-CT fusion is needed. The image registration of TT CT and CT after BT treatment does not use the dose values from the real BT plan. The dose gradient is high in BT, so the dose distribution can be significantly different in a post-BT plan without the needles and US probe than in the actual plan. Using doses of the actual plan, where the needles are in their real place, is the most adequate method.

We have developed an alternative dose summation method in combined radiotherapy of cervical cancer [14]. The aim of the present study was to elaborate on alternative method for summing the biologically effective doses of IMAT TT with interstitial HDR-BT boost in prostate cancer and compare it to the recent UDC method. Additionally, the total doses of IMAT TT plus HDR-BT vs. IMAT TT boost will also be compared using our dose summation method.

\section{Material and methods}

At our institute, twenty-five IMAT TT plus interstitial HDR-BT plans of patients with intermediate- and high-risk prostate cancer were included for this study. Selection criteria included prostate-specific antigen (PSA) $>10 \mathrm{ng} / \mathrm{ml}$, and/or Gleason score (GS) 7-10 and/or stage T2b-T3b. The TT was performed in supine position, the patients were immobilized with knee and ankle support system. The prescribed dose was 2/44 Gy for the whole pelvis, 2/60 Gy for the prostate and the seminal vesicles, and was delivered with an energy of $10 \mathrm{MV}$, using 2 full arcs. Based on our local IGRT protocol, CBCT verification was made from $1^{\text {st }}$ to $3^{\text {rd }}$ fractions, the systematic error was calculated and corrected before the $4^{\text {th }}$ fraction, then weekly verification was done for patients' positioning. TT was complemented with transrectal US-guided interstitial HDR-BT boost, performed after 4 weeks of TT course, given 1 fraction of 10 Gy [19]. After scanning the prostate with US, a virtual preimplant plan was generated (Oncentra Prostate v3.1, Elekta Brachytherapy, Veendendaal, The Netherlands). The whole prostate gland was contoured as the target volume, and OARs included the rectum (rectal wall), the prostatic-urethra, and the bladder. HIPO optimization method was used, and the prescribed dose was $10 \mathrm{~Gy}$ to the whole prostate gland $\left(V_{100} \geq 95 \%\right)$. Based on this plan, metal needles were inserted into the prostate through a template under US guidance. The optimization procedure was used again for calculating dwell times in the inserted needles to achieve final dose distribution. The detailed description of our treatment method can be found in our previous publications $[20,21]$. The total treatment time of TT and BT was 7 weeks (range, 44-54 days). In clinical routine, the UDC method was used to determine the dose constraints for prostate and OARs in BT implant and their total doses.

First, the treatment planning CT for TT was registered with the US BT set in BT treatment planning system in every case (Figure 1). During the manual registration, the TT CT set was shifted and rotated to place together the prostate gland of BT and TT plans, and rectal walls of the two plans. Then, the TT CT with BT plan was imported to the TT planning system (Eclipse v13.7, Varian Medical Systems, Palo Alto, USA).

Then, the localization of the most exposed part of OARs from the sum of TT and BT plans was investigated. The most exposed part of hips (femoral heads) is always the nearest volume to the prostate and the dose contribution from BT is practically zero. Therefore, the most 
A

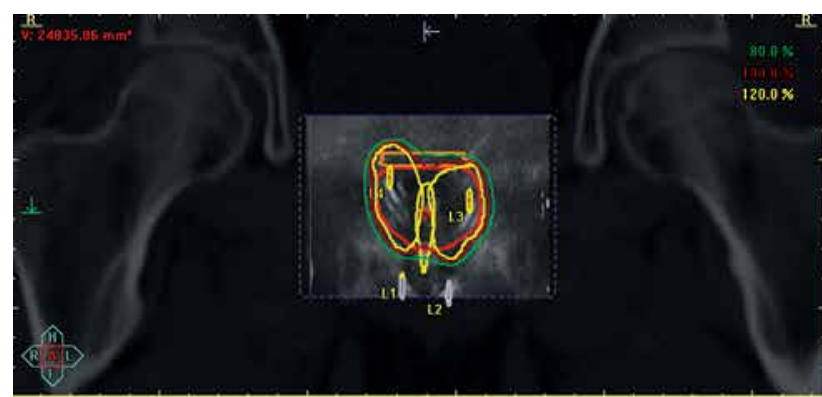

C

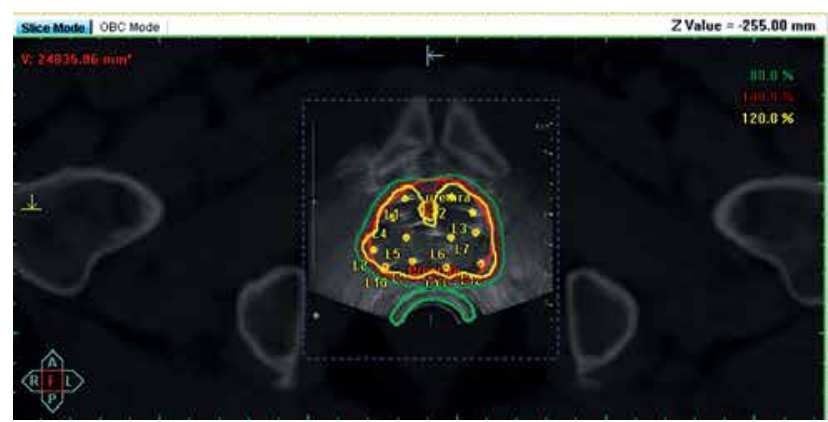

B

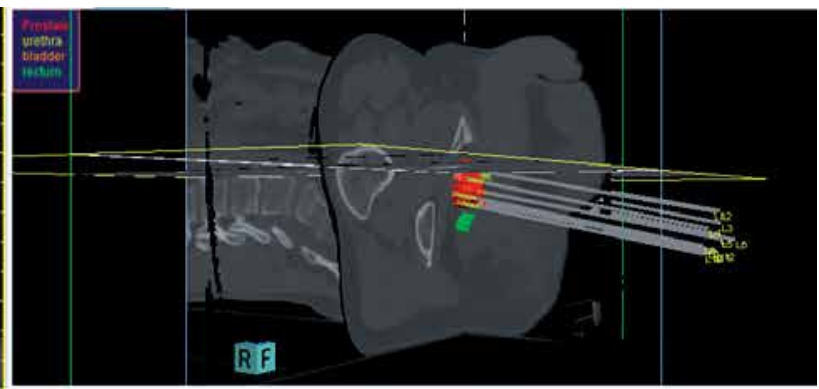

D

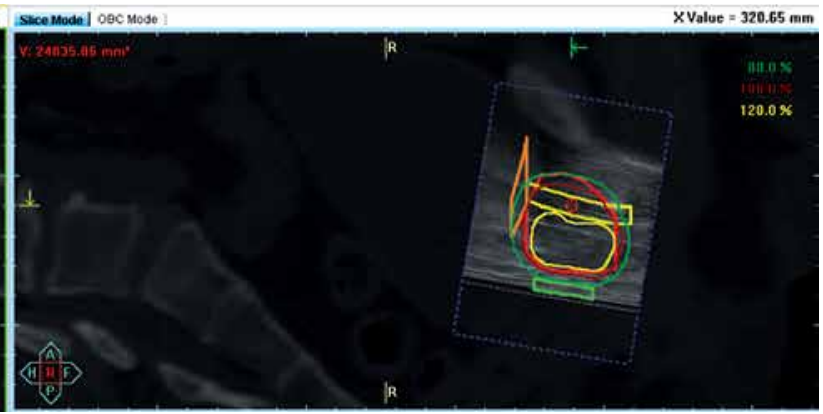

Fig. 1. The BT treatment plan on the registered TT CT and BT US sets. A) A coronal view; B) 3D reconstruction; C) An axial view; D) A sagittal view. Thick red - prostate, thick green - rectum, thick yellow - urethra, thick orange - bladder, green, red, and yellow line - the $80 \%, 100 \%$, and $120 \%$ isodose line

exposed 0.1 and $2 \mathrm{~cm}^{3}$ of hips were calculated only from the TT plan. The most exposed part of the rectum, urethra, and bladder is in the region where the dose maximum is in BT. So, the most exposed 0.1 and $2 \mathrm{~cm}^{3}$ from BT were determined in the TT CTs, and the intersection of this volumes and given organ was created (Figure 2). The minimal dose of this intersection was calculated in TT plans and summed with the dose of this volumes from BT using the linear-quadratic radiobiological model. The $\alpha / \beta$ of prostate tumor was assumed as $1.5 \mathrm{~Gy}[22,23,24]$, while for OARs, 3 Gy was used. The minimum dose delivered to $90 \%$ of the prostate $\left(\mathrm{D}_{90}\right)$ was calculated in the TT and BT plans, and these doses were summed using also the linear-quadratic model.

The following dose-volume parameters were used for quantitative evaluation of the plans: $D_{90}$ - the minimum dose delivered to $90 \%$ of prostate $(\mathrm{Gy}), \mathrm{D}_{0.1}(\mathrm{x})$ - the minimal dose of the most exposed $0.1 \mathrm{~cm}^{3}$ of the critical organ $x(G y)$, where $x$ were the rectum, urethra, bladder, or hips. $\mathrm{D}_{2}(\mathrm{x})$ - the minimal dose of the most exposed $2 \mathrm{~cm}^{3}$ of critical organ $x(G y)$, where $x$ were rectum, bladder, or hips.

In patients with not accomplishable BT, TT boost is performed with additional $18 \mathrm{~Gy}$ in $2 \mathrm{~Gy}$ fractions for the prostate gland, using a uniform CTV $\rightarrow$ PTV expansion margin of $0.5 \mathrm{~cm}$ if gold markers are implanted into the prostate and $0.8 \mathrm{~cm}$ if are not implanted [25,26]. For comparison, additional TT boost plans were created for every patient in the study with the same IMAT technique, and the total $\mathrm{EQD}_{2}$ doses of the most exposed volume of organs at risks were calculated in these 3-step TT plans.

Wilcoxon-matched pairs test was used (Statistica 12.5, StatSoft, Tulsa, OK, USA) to compare biological total dose of the combination of TT and BT or TT boost in the treatment of prostate tumor. The comparison of our biological dose summation (BDS) and conventional UDC method was also performed with this statistical test.

\section{Results}

The mean prostate volume was $29.8 \mathrm{~cm}^{3}$ (range, 21.1$43.0 \mathrm{~cm}^{3}$ ). We found that $\mathrm{EQD}_{2} \mathrm{D}_{90}$ of the prostate was 99.3 Gy (range, 96.8-101.9 Gy) using two-step TT and BT boost. The $\mathrm{D}_{0.1}$ and $\mathrm{D}_{2}$ of rectum were $62.8 \mathrm{~Gy}$ (range, 41.0-75.6 Gy) and 50.3 Gy (range, 29.8-65.8 Gy). The $\mathrm{D}_{0.1}$ of urethra was $96.1 \mathrm{~Gy}$ (range, 95.5-96.9 Gy), with the volume less than $2 \mathrm{~cm}^{3}$ in our cases. The $\mathrm{D}_{0.1}$ and $\mathrm{D}_{2}$ of bladder were 75.4 Gy (range, 62.5-92.9 Gy) and 64.7 Gy (range, 46.0-73.8 Gy). The $\mathrm{D}_{0.1}$ and $\mathrm{D}_{2}$ of hips were $49.6 \mathrm{~Gy}$ (range, 39.8-67.3 Gy) and 41.9 Gy (range, 33.5-58.3 Gy).

In TT boost, the volume of PTV was larger than the prostate and was $111.7 \mathrm{~cm}^{3}$ on average (range, $71.9-179.5 \mathrm{~cm}^{3}$ ). Comparing BT and TT boost, the $\mathrm{D}_{90}$ of prostate was significantly higher with BT than with TT: 99.3 Gy vs. 77.9 Gy, $p=0.0034$. The dose to rectum, bladder, and hips were significantly lower with $\mathrm{BT}$ boost, $\mathrm{D}_{2}$ was 50.3 Gy vs. 76.8 Gy $(p=0.0117), 64.7$ Gy vs. $78.3 \mathrm{~Gy}(p=0.0117)$, and 41.9 Gy vs. 50.6 Gy $(p=0.0044)$, respectively. Nevertheless, the dose to urethra was significantly higher with BT boost, $\mathrm{D}_{0.1}$ was $96.1 \mathrm{~Gy}$ vs. $79.3 \mathrm{~Gy}(p=0.0180)$ using BT vs. TT boost technique (Figure 3 ). The detailed results are presented in Table 1.

Comparing our dose summation method to the conventional UDC in combined TT and BT boost, we found that the UDC overestimated $\mathrm{D}_{2}$ of rectum by $37 \%$ 


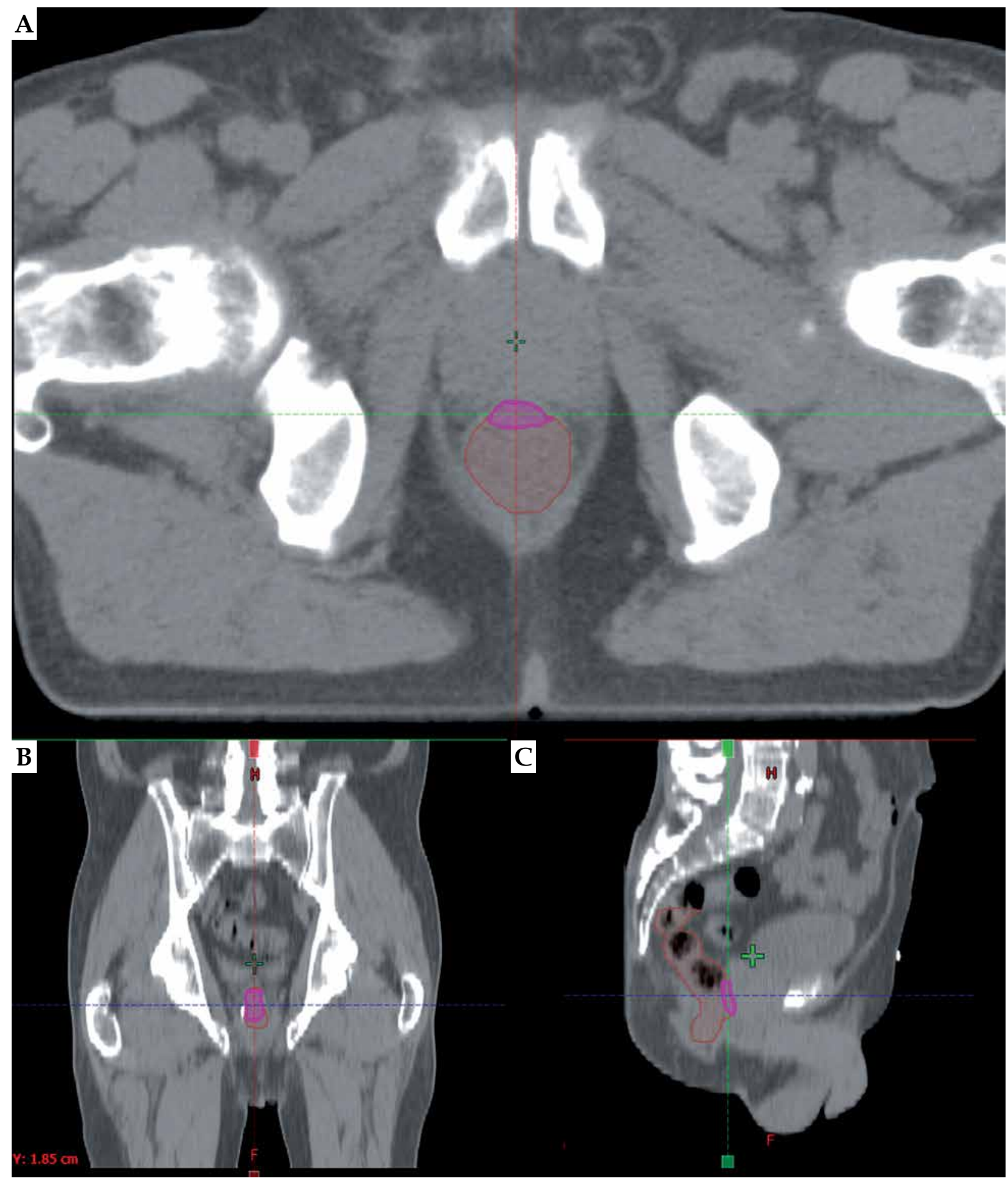

Fig. 2. The most exposed $2 \mathrm{~cm}^{3}$ part (pink) of rectum (brown) in axial (A), in coronal (B), and in sagittal (C) slice of the TT CT

( $p=0.0117), \mathrm{D}_{2}$ of bladder by $5 \%(p=0.0214)$, and underestimated $\mathrm{D}_{0.1}$ of urethra by $1 \%(p=0.0277)$ (Table 2$)$.

\section{Discussion}

Dose escalation has a fundamental role in radiotherapy of intermediate- and high-risk prostate cancer [1,2] Presently, there are no better alternatives of BT boost; however, several high-tech teletherapy techniques are possible competitors, such as image-guided and intensitymodulated teletherapy, arc therapy, helical tomotherapy, and stereotactic radiotherapy with linear accelerators or CyberKnife $[3,7,16]$.

Vanneste et al. [1] reported a strong correlation between overall survival and $\mathrm{D}_{90}$ of prostate target volume in localized prostate cancer, with the best results being 


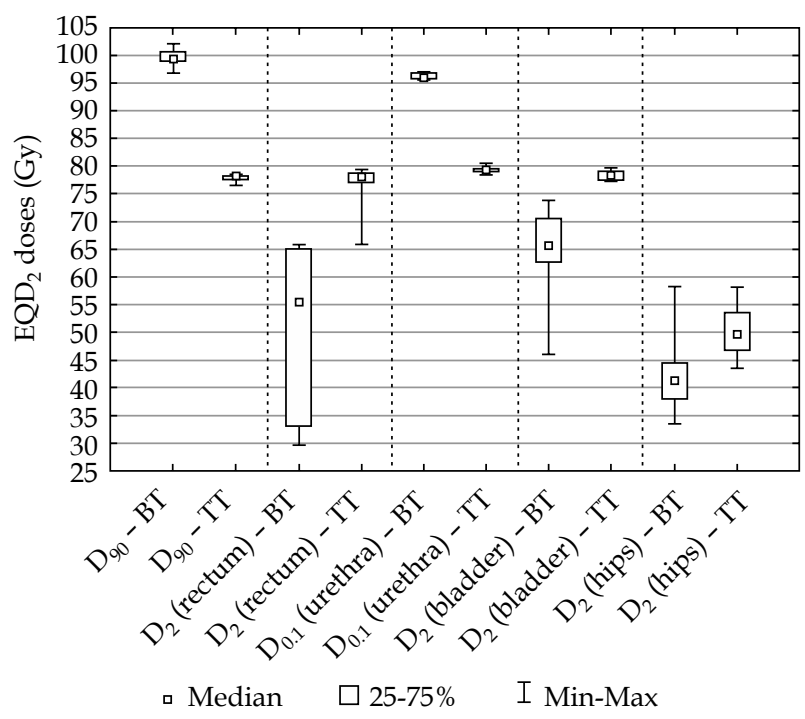

Fig. 3. The $\mathrm{EQD}_{2}$ total doses of intensity-modulated arc therapy plus interstitial HDR-BT boost (BT), and intensitymodulated arc therapy plus teletherapy boost (TT)

$D_{90}$ - minimum dose delivered to $90 \%$ of the prostate (Gy), $D_{2}$ (rectum), $D_{2}$ (bladder), $D_{2}$ (hips) - minimal dose of the most exposed $2 \mathrm{~cm}^{3}$ of rectum, bladder, and hips (Gy), $D_{0.1}$ (urethra) - minimal dose of the most exposed $0.1 \mathrm{~cm}^{3}$ of urethra (Gy)

achievable above 75.6 Gy EQD 2 . Different treatment techniques showed similar curative rates, but with different toxicity patterns. The $\mathrm{EQD}_{2}$ prescribed dose to the prostate with our fractionation scheme is 92.9 Gy using BT and 78 Gy with TT boost. At the same time, the dose to OARs is reduced with BT [3,4]. In our study, the use of IMAT TT with HDR-BT boost could keep the dose of all OARs under the tolerance level. The $\mathrm{EQD}_{2} \mathrm{D}_{90}$ of the prostate was $99.3 \mathrm{~Gy}$, while $\mathrm{D}_{2}$ of the rectum was $50.3 \mathrm{~Gy}$, approximately half of the prostate dose. $\mathrm{D}_{0.1}$ dose to the urethra was $96.1 \mathrm{~Gy}$ on average, less than the prostate dose, despite the urethra being inside the prostate. $D_{2}$ dose to the bladder was $64.7 \mathrm{~Gy}$, while for the hips, it was only 41.9 Gy. All dose to the hips originated from 60 Gy of TT, BT did not contribute to the dose.

Even though in TT larger target volume is used than in $\mathrm{BT}$, the total dose to the prostate was $22 \%$ less (21.4 Gy), $\mathrm{D}_{90}$ was $99.3 \mathrm{~Gy}$ using BT and 77.9 Gy with TT boost. $\mathrm{D}_{2}$ dose to the rectum, bladder, and hips were 35\% (26.5 Gy), 17\% (13.6 Gy), and 18\% (8.7 Gy) lesser with BT than using TT boost. 18 Gy IMAT TT boost to the prostate target volume instead of BT means extra 9 Gy dose to the hips. Only the dose to the urethra was higher with $\mathrm{BT}$ boost, $\mathrm{D}_{0.1}$ was 18\% (16.8 Gy) higher than using TT boost. Pieters et al. [17] concluded the same tendency in case of bladder, rectum, and urethra for low-risk patients, where the target volume was only the prostate, and the total $\mathrm{EQD}_{2}$ was 76 Gy using TT boost and 70 Gy using BT boost. The higher dose to the urethra using BT boost can account for more serious urethral toxicity in case of BT than TT boost.

In previous publications, authors used the recommended UDC method to estimate the total dose of prostate and OARs in a combined therapy [15]. However, they did not consider the real biological doses. Kikuchi et al. [18] examined a better estimation of rectal dose than the UDC method, but they used a CT after removing the needles and US probe instead of a post-implant CT or actual US imaging in the intraoperative BT plan, and they did not take into account the quadratic behavior of biological dose. Since the most exposed part of

Table 1. The $\mathrm{EQD}_{2}$ total doses of intensity-modulated arc therapy plus interstitial HDR-BT boost (TT + BT boost) and intensity-modulated arc therapy plus teletherapy boost (TT + TT boost)

\begin{tabular}{llll}
$\mathrm{EQD}_{2}$ & $\mathrm{TT}+\mathrm{BT}$ boost & TT + TT boost & $P$-value \\
\hline $\mathrm{D}_{90}$ (Gy) & $99.3(96.8-101.9)$ & $77.9(76.4-78.5)$ & 0.0034 \\
\hline $\mathrm{D}_{2}$ (rectum) (Gy) & $50.3(29.8-65.8)$ & $76.8(65.8-79.3)$ & 0.0017 \\
\hline $\mathrm{D}_{0.1}$ (urethra) (Gy) & $96.1(95.5-96.9)$ & $79.3(78.6-80.4)$ & 0.0180 \\
\hline $\mathrm{D}_{2}$ (bladder) (Gy) & $64.7(46.0-73.8)$ & $78.3(77.2-79.8)$ & 0.0117 \\
\hline $\mathrm{D}_{2}$ (hips) (Gy) & $41.9(33.5-58.3)$ & $50.6(43.6-58.1)$ & 0.0044
\end{tabular}

$D_{90}$ - minimum dose delivered to $90 \%$ of the prostate $(G y), D_{2}$ (rectum), $D_{2}$ (bladder), $D_{2}$ (hips) - minimal dose of the most exposed $2 \mathrm{~cm}^{3}$ of rectum, bladder, and hips (Gy), $D_{0.1}$ (urethra) - minimal dose of the most exposed $0.1 \mathrm{~cm}^{3}$ of urethra (Gy), *Wilcoxon-matched pairs test

Table 2. The $\mathrm{EQD}_{2}$ total doses of intensity-modulated arc therapy plus interstitial HDR-BT boost calculated by our biological dose summation (BDS) and the uniform dose conception (UDC) method

\begin{tabular}{lccc}
$\mathrm{EQD}_{2}$ & BDS & UDC & $P$-value* \\
\hline $\mathrm{D}_{90}$ (Gy) & $99.3(96.8-101.9)$ & $100.2(96.6-104.8)$ & 1.0000 \\
\hline $\mathrm{D}_{2}$ (rectum) (Gy) & $50.3(29.8-65.8)$ & $68.9(66.6-70.9)$ & 0.0117 \\
\hline $\mathrm{D}_{0.1}$ (urethra) (Gy) & $96.1(95.5-96.9)$ & $95.4(94.4-96.0)$ & 0.0277 \\
\hline $\mathrm{D}_{2}$ (bladder) (Gy) & $64.7(46.0-73.8)$ & $68.2(62.9-74.0)$ & 0.0214
\end{tabular}

$D_{90}$ - minimum dose delivered to $90 \%$ of the prostate (Gy), $D_{2}$ (rectum), $D_{2}$ (bladder) - minimal dose of the most exposed $2 \mathrm{~cm}^{3}$ of rectum and bladder (Gy), $D_{0.1}$ (urethra) - minimal dose of the most exposed $0.1 \mathrm{~cm}^{3}$ of urethra (Gy), *Wilcoxon-matched pairs test 

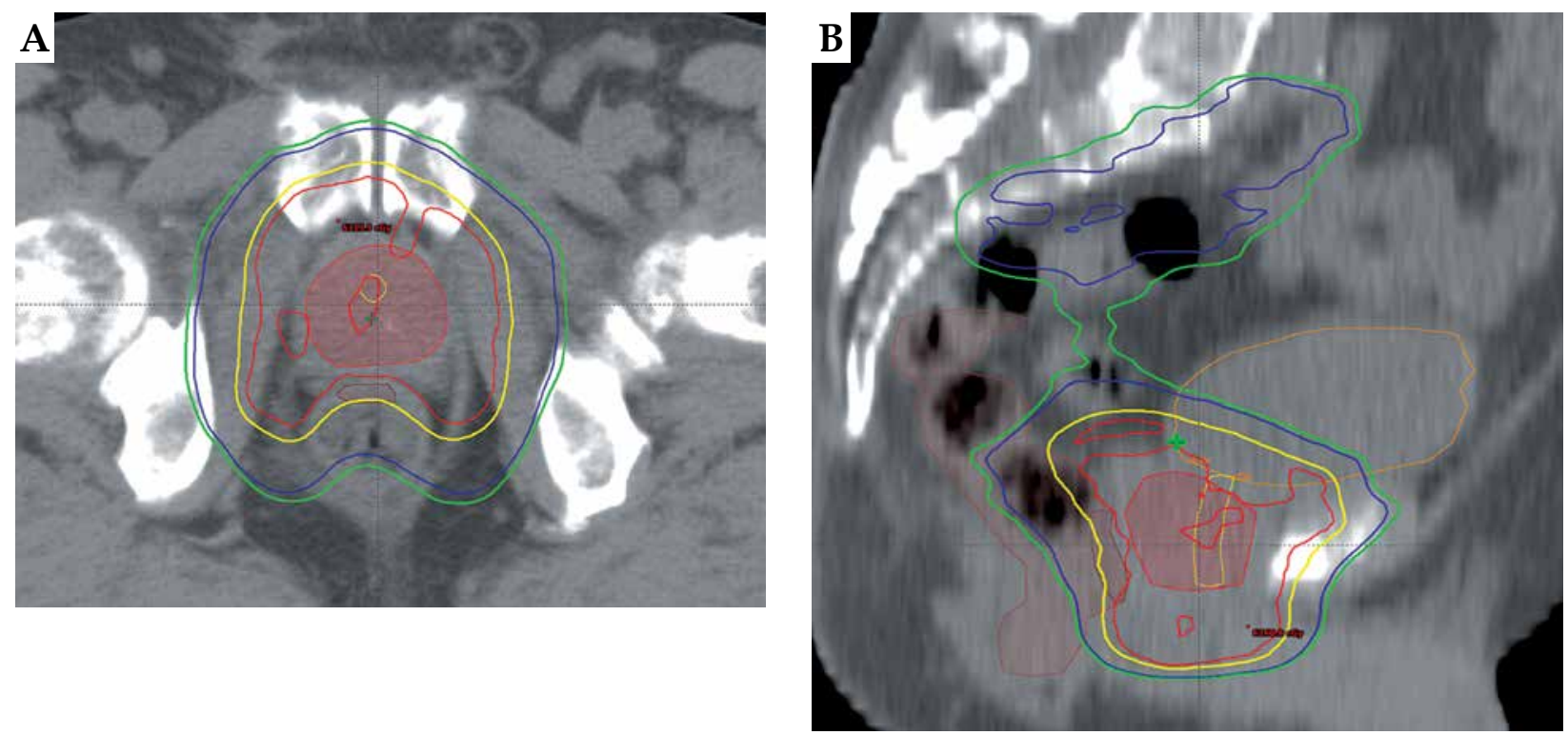

Fig. 4. The most exposed $2 \mathrm{~cm}^{3}$ of rectum is indicated with brown, the urethra and the bladder are contoured with yellow and orange, and the prostate gland is shown with red (color wash) in axial (A) and sagittal (B) CT slice in two-step intensity-modulated arc therapy plan. Isodose lines: red - $60 \mathrm{~Gy}$, yellow - $57 \mathrm{~Gy}$, blue - $44 \mathrm{~Gy}$, and green - 41.8 Gy

the rectum, urethra, and bladder is the region, where the dose maximum is in BT, this most exposed $2 \mathrm{~cm}^{3}$ can be used for the calculation of the total biological dose. In this small volume, the quadratic dependence is negligible. Thus, our dose summation method is simple, timesaving, and more personalized than the EUD method. The only more precise method would be the pixel-by-pixel calculation of biological dose in the same organ after a deformable registration of BT and TT images, but no treatment planning systems provides this possibility at present.

The effect of dose summation technique on dose-volume parameters in combined TT and BT was also investigated in our study. The EQD $\mathrm{D}_{90}$ of the prostate was practically identical in our BDS and the conventional UDC method, but UDC overestimated the dose to rectum by $37 \%$ (18.6 Gy), dose to bladder by $5 \%$ (3.5 Gy), and underestimated the dose to urethra by $1 \%$ (0.7 Gy) as compared to the BDS method. The cause may be that the dose of critical organs can be decreased with the IMAT technique, so the most exposed volumes of these organs can be irradiated with lower than the prescribed dose. Accordingly, the potential advantage of BDS method is that it considers the most exposed part of OARs and thus sparing these parts from higher doses in TT, as shown in Figure 4. Overall, the dose to OARs can be reduced using our alternative dose summation method, therefore the treatment-related toxicity can be decreased.

This study is the starting point of the development of an algorithm for the summation of TT and BT biologically effective doses, which uses an artificial intelligence-based DIR algorithm to match the critical anatomical structures in the two radiotherapy modalities. Further investigations are needed to assess whether our method predicts toxicity better than the recent UDC method.

\section{Conclusions}

Based on our biological dose summation method in IMAT TT with interstitial HDR-BT or IMAT TT boost treatment in prostate cancer, the total dose of the prostate is higher using BT boost than the TT. BT boost results in lower rectum, bladder, and hip doses, but higher dose to the urethra. UDC overestimates the rectum and bladder dose, and underestimates the dose to urethra, as compared to our method.

\section{Disclosure}

This paper was supported by the János Bolyai Research Scholarship of the Hungarian Academy of Sciences and the ÚNKP-18-4 New National Excellence Program of the Ministry of Human Capacities.

The authors report no conflict of interest.

\section{References}

1. Vanneste BG, Van Limbergen EJ, van Lin EN et al. Prostate cancer radiation therapy: what do clinicians have to know? Biomed Res Int 2016; 2016: 6829875.

2. Kuban DA, Tucker SL, Dong YL et al. Long-term results of the M. D. Anderson randomized dose-escalation trial for prostate cancer. Int J Radiat Oncol Biol Phys 2008; 70: 67-74.

3. Georg D, Hopfgartner J, Gòra J et al. Dosimetric considerations to determine the optimal technique for localized prostate cancer among external photon, proton, or carbon-ion therapy and high-dose-rate or low-dose-rate brachytherapy. Int J Radiat Oncol Biol Phys 2014; 188: 715-722.

4. Yang R, Zhao N, Liao A et al. Dosimetric and radiobiological comparison of volumetric modulated arc therapy, high-dose rate brachytherapy, and low-dose rate permanent seeds implant for localized prostate cancer. Med Dosim 2016; 41: 236-241.

5. Kee DLC, Gal J, Falk AT et al. Brachytherapy versus external beam radiotherapy boost for prostate cancer: Systematic review with meta-analysis of randomized trials. Cancer Treat Rev 2018; 70: 265-271. 
6. Fu-Min F, Yu-Ming W, Chong-Jong W et al. Comparison of the outcome and morbidity for localized or locally advanced prostate cancer treated by high-dose-rate brachytherapy plus external beam radiotherapy (EBRT) versus EBRT alone. Jpn J Clin Oncol 2008; 38: 474-479.

7. Morgan TM, Press RH, Cutrell PK et al. Brachytherapy for localized prostate cancer in the modern era: a comparison of patient-reported quality of life outcomes among different techniques. J Contemp Brachytherapy 2018; 10: 495-502.

8. Ash D, Al-Qaisieh B, Bottomley D et al. The correlation between D90 and outcome for I-125 seed implant monotherapy for localised prostate cancer. Radiother Oncol 2006; 79: 185-189.

9. Murakami N, Itami J, Okuma K et al. Urethral dose and increment of international prostate symptom score (IPSS) in transperineal permanent interstitial implant (TPI) of prostate cancer. Strahlenther Onkol 2008; 184: 515-519.

10. Fowler JF. The linear-quadratic formula on progress in fractionated radiotherapy. Br J Radiol 1989; 62: 679-694.

11. Nag S, Gupta N. A simple method of obtaining equivalent doses for use in HDR brachytherapy. Int J Radial Oncol Biol Phys 2000; 46: 507-513.

12. Niemierko A. Reporting and analyzing dose distributions: a concept of equivalent uniform dose. Med Phys 1997; 24: 103110.

13. Fröhlich G, Lang S, Berger D et al. Spatial relationship of the 3D dose distribution from brachytherapy and external beam therapy for adding both dose plans in patients with cervix cancer. Brachytherapy 2008; 7: 95.

14. Fröhlich G, Vízkeleti J, Nguyen AN et al. Comparative analysis of image-guided adaptive interstitial brachytherapy and intensity-modulated arc therapy versus conventional treatment techniques in cervix cancer using biological dose summation. J Contemp Brachytherapy 2019; 11: 69-75.

15. Pinkawa M, Fischedick K, Treusacherr P et al. Dose-volume impact in high-dose-rate Iridium-192 brachytherapy as a boost to external beam radiotherapy for localized prostate cancer- a phase II study. Radiother Oncol 2006; 78: 41-46.

16. Andrzejewski P, Kuess P, Knäusl B et al. Feasibility of dominant intraprostatic lesion boosting using advanced photon-, proton- or brachytherapy. Radiother Oncol 2015; 117: 509-514.

17. Pieters BR, van de Kamer JB, van Herten YR et al. Comparison of biologically equivalent dose-volume parameters for the treatment of prostate cancer with concomitant boost IMRT versus IMRT combined with brachytherapy. Radiother Oncol 2008; 88: 46-52.

18. Kikuchi K, Nakamura R, Tanji $\mathrm{S}$ et al. Three-dimensional summation of rectal doses in brachytherapy combined with external beam radiotherapy for prostate cancer. Radiother $\mathrm{On}$ col 2013; 107: 159-164.

19. Kovács G, Pötter R, Loch T et al. GEC/ESTRO-EAU recommendations on temporary brachytherapy using stepping sources for localised prostate cancer. Radiother Oncol 2005; 74: $137-148$.

20. Fröhlich G, Ágoston P, Lövey J et al. Dosimetric evaluation of high-dose-rate interstitial brachytherapy boost treatments for localized prostate cancer. Strahlenther Onkol 2010; 186: 388-395.

21. Ágoston P, Major T, Fröhlich G et al. Moderate dose escalation with single-fraction high-dose-rate brachytherapy boost for clinically localized intermediate- and high-risk prostate cancer: 5-year outcome of the first 100 consecutively treated patients. Brachytherapy 2011; 10: 376-384.

22. Dasu A, Toma-Dasu I. Prostate alpha/beta revisited - an analysis of clinical results from 14168 patients. Act Oncol 2012; 51: 963-974.

23. Leborgne F, Fowler J, Jos Y et al. Later outcomes and alpha/ beta estimate from hypofractionated conformal three-di- mensional radiotherapy versus standard fractionation for localized prostate cancer. Int J Radiat Oncol Biol Phys 2012; 82: 1200-1207.

24. Vogelius IR, Bentzen SM. Meta-analysis of the alpha/beta ratio for prostate cancer in the presence of an overall time factor: bad news, good news, or no news? Int J Radiat Oncol Biol Phys 2013; 85: 89-94.

25. Boehmer D, Maingon P, Poortmans P et al. EORTC radiation oncology group. Guidelines for primary radiotherapy of patients with prostate cancer. Radiother Oncol 2006; 79: 259-269.

26. Lawton CA, Michalski J, El-Naqa I et al. RTOG GU Radiation oncology specialists reach consensus on pelvic lymph node volumes for high-risk prostate cancer. Int J Radiat Oncol Biol Phys 2009; 74: 383-387. 